\title{
List of Contributors
}

\section{Amit Adate}

School of Computer Science and Engineering Vellore Institute of Technology

Vellore, Tamil Nadu, India

email2amitadate@gmail.com

\section{Dhruv Arya}

School of Computer Science and Engineering Vellore Institute of Technology

Vellore, Tamil Nadu, India

aryadhruv@gmail.com

\section{Siddhartha Bhattacharyya}

CHRIST (Deemed to be University)

Bangalore, Karnataka, India

dr.siddhartha.bhattacharyya@gmail.com

\section{Ankita Bose}

VIT University, Vellore, Tamil Nadu, India a1997bose@gmail.com

\section{Mahua Bose}

Department of Computer Science and Engineering, University of Kalyani Nadia, West Bengal, India

e_cithi@yahoo.com

\section{Swagatam Das}

Indian Statistical Institute

Kolkata, West Bengal, India

swagatamdas19@yahoo.co.in

\section{Shatabhisa Dey}

RCC Institute of Information Technology

Kolkata, West Bengal, India

shatabhisa.97@gmail.com

\section{Soumyajit Goswami}

IBM India Private Limited, Salt Lake, Sector V

Kolkata 700091, West Bengal, India

soumyajit_goswami@yahoo.com

\section{Ranjan Jana}

RCC Institute of Information Technology

Kolkata, West Bengal, India

ranjan.rcciit@gmail.com

\section{Karan Maheshwari}

School of Computer Science and Engineering

Vellore Institute of Technology

Vellore, Tamil Nadu, India

karan@cognet.ai

\section{Kalyani Mali}

Department of Computer Science and

Engineering, University of Kalyani

Nadia, West Bengal, India

kalyanimali1992@gmail.com

\section{Anirban Mukherjee}

RCC Institute of Information Technology

Kolkata, West Bengal, India

anirbanm.rcciit@gmail.com

\section{Rajib Saha}

RCC Institute of Information Technology

Kolkata, West Bengal, India

rajibsaha_4u@yahoo.co.in

\section{Aditya Shaha}

School of Computer Science and Engineering Vellore Institute of Technology

Vellore, Tamil Nadu, India

aditya.shaha.p@gmail.com

\section{Rajkumar Rajasekaran}

School of Computer Science and Engineering Vellore Institute of Technology

Vellore, Tamil Nadu, India

rrajkumar@vit.ac.in

\section{Avik Sarkar}

RCC Institute of Information Technology

Kolkata, West Bengal, India

avik.ron20@gmail.com

\section{B. K. Tripathy}

School of Information Technology and Engineering, Vellore Institute of Technology Vellore, Tamil Nadu, India

tripathybk@rediffmail.com 
